Weronika Urbanik-Pęk

Uniwersytet Jagielloński weronika.urbanik@uj.edu.pl

\title{
Asimilación fonética anticipatoria de las consonantes nasales en los vocablos polacos y españoles
}

\begin{abstract}
:
Regressive Assimilation of Nasals in Polish and Spanish Sound Systems The article concerns the phenomenon of regressive assimilation of nasals in Polish and Spanish sound systems. The basic information is followed by the description of the examples of the assimilations which involve the change of place of articulation in respective languages. The author shows the differences in the articulation of particular sounds and emphasizes the peculiarity of Polish in which the hard and soft and voiced and voiceless classifications of consonants can be observed. Polish also uses nasal vowels whose phonetic realization is similar to that of nasal consonants.
\end{abstract}

Keywords: assimilation, nasal consonants

\section{Streszczenie:}

Upodobnienia wsteczne w systemie spólgłosek nosowych w języku polskim i hiszpańskim

Niniejszy artykuł porusza zagadnienie upodobnień wstecznych w systemie spółgłosek nosowych w języku hiszpańskim i polskim. Po przybliżeniu podstawowych informacji, zostały opisane asymilacje, które zachodzą w jed- 
nym i drugim języku, zmieniając miejsce artykulacji danej spółgłoski nosowej. Artykuł prezentuje różnice w możliwości artykulacji poszczególnych głosek, akcentując osobliwość języka polskiego, który rozróżnia spółgłoski twarde i miękkie, dzieląc je ponadto na dźwięczne i bezdźwięczne, a także dysponuje samogłoskami nosowymi, których realizacja fonetyczna jest często podobna do realizacji spółgłosek nosowych.

Słowa kluczowe: upodobnienie, spółgłoski nosowe, asymilacja

\section{Introducción}

La pretensión del presente artículo es poner de relieve la cuestión del cambio fonético de las consonantes nasales. Las nasales son consonantes que se producen por el cierre completo de la cavidad oral pero con el velo del paladar caído, de modo que el paso del aire se produce a través de la cavidad nasal. En este artículo serán comparados los sistemas polaco y español de las consonantes nasales, y con posterioridad se analizarán asimilaciones fonéticas que se producen en ambos sistemas. La fonología actual extiende su dominio de estudio a los procesos que tienen lugar en la realización de los segmentos en la cadena hablada porque, con el paso del tiempo, la articulación de ciertos sonidos cambia. Como sabemos, la pronunciación de un segmento de lengua varía dependiendo de la presencia de otro adyacente o cercano. Los procesos que afectan a los segmentos del español pueden clasificarse en cuatro grandes categorías: procesos que exigen un refuerzo en la pronunciación de determinados segmentos; procesos de debilitamiento, que pueden desembocar en la elisión de una unidad; procesos de disimilación, que tienen como objetivo la diferenciación de unidades similares y, finalmente, procesos de asimilación. Mediante los últimos, que constituyen nuestro centro de interés, "un segmento se disocia de alguno de sus rasgos y adopta rasgos de un segmento contiguo, con lo que se produce, a su vez, un proceso de asociación de rasgos distintivos" Blecua, 2011: 270]. Dependiendo del modo de asimilación, se pueden distinguir asimilaciones que se 
dan en condiciones de adyacencia o de cercanía. Pueden producirse respecto al fonema anterior, siendo entonces asimilación progresiva, o al fonema posterior, siendo asimilación regresiva.

En el presente artículo nos fijaremos en las asimilaciones regresivas (o anticipatorias) por adyacencia, que consisten en la articulación de un fonema con rasgos fonéticos del fonema inmediatamente posterior. Se han elegido éstas puesto que en dicho grupo podemos observar más cambios fonéticos en cuanto a las nasales, tanto en polaco como en español. Los siguientes capítulos ofrecerán una variada información sobre las consonantes nasales en español y sus asimilaciones, describiéndose a continuación el sistema de las nasales en polaco y sus cambios fonéticos dentro de palabras concretas. Posteriormente, otra parte del artículo se dedicará a la comparación de ambos sistemas y de las asimilaciones que se producen en estas dos lenguas, indicando diversas similitudes y subrayando posibles discrepancias, concluyéndose, para finalizar, con unas reflexiones acerca de todo el contenido previamente presentado.

\section{Las consonantes nasales en español}

De acuerdo con la Nueva gramática de la lengua española. Fonética y fonología, el español presenta tres consonantes nasales: $/ \mathrm{m} /, / \mathrm{n} / \mathrm{y}$ $/ \mathrm{y} /$. Se diferencian entre sí por los rasgos relacionados con la zona o lugar de articulación:

\begin{tabular}{|c|c|c|c|}
\cline { 2 - 4 } \multicolumn{1}{c|}{} & bilabial & alveolar & palatal \\
\hline $\begin{array}{c}\text { sonantes } \\
\text { nasales }\end{array}$ & $\mathrm{m}$ & $\mathrm{n}$ & $\mathrm{n}$ \\
\hline
\end{tabular}

Tabla 1. Consonantes nasales en españolNo obstante cabe enumerar también diversas realizaciones de las nasales antes mencionadas. Según E. Martínez Celdrán y A.M. Fernández Planas, las realizaciones de las nasales son las siguientes. 


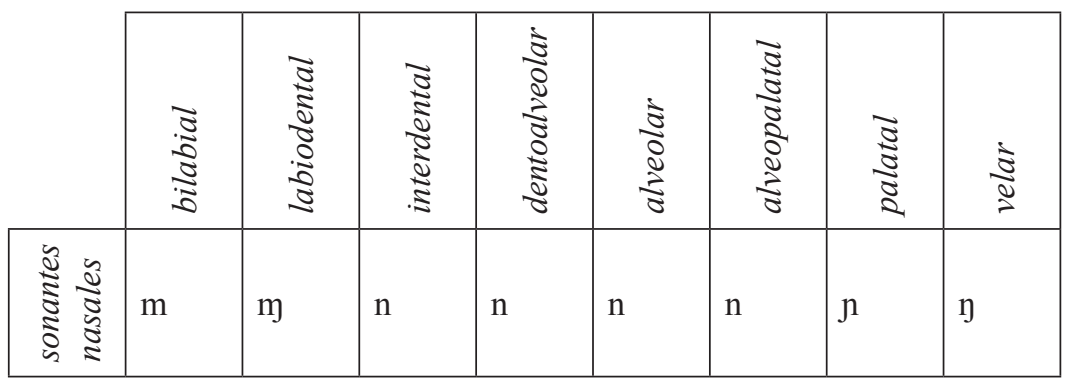

Tabla 2. Fragmento del cuadro de sonidos del español. Realizaciones de las consonantes nasales en español [Martínez Celdrán, Fernández Planas, 2007: 237]. Dichas realizaciones de las nasales se producen por influencia de otros segmentos de la lengua que modifican la zona o lugar de articulación y su posición en la sílaba. En posición postnuclear, las consonantes $/ \mathrm{m} /, / \mathrm{n} /, / \mathrm{n} /$ pierden los rasgos por los que contrastan y presentan diferentes alófonos, caracterizados en parte por el mantenimiento de la resonancia nasal y de la oclusión bucal, así como por la asimilación o asociación de su zona articulatoria a los rasgos de la consonante siguiente. Estas variaciones originan las demás realizaciones.

\section{Asimilaciones regresivas de las consonantes nasales en español}

En este apartado serán analizados, paso a paso, todos los procesos que producen un cambio en la realización de las nasales. La variante bilabial $/ \mathrm{m} /$ aparece ante otras bilabiales, como lo son $/ \mathrm{p} / \mathrm{y} / \mathrm{b} /$ : esto puede observarse, por ejemplo, en las palabras embarazo, empanada, tan bien. Es posible apreciar en el último ejemplo que la letra que aparece no es la $m$ sino la $n$, aunque en esa posición es pronunciada como una $/ \mathrm{m} /$ bilabial. El proceso aquí descrito se llama bilabialización [Blecua, 2011: 278].

Otra realización de la nasal bilabial $/ \mathrm{m} /$ es la labiodental $/ \mathrm{m} /$, que se da normalmente ante la fricativa /f/, por ejemplo en: enfermera o énfasis. Cabe mencionar que en este caso también encontramos la 
grafía $n$, que en la pronunciación ante una fricativa labiodental, pierde su punto de articulación, asimilándose a la zona de articulación de la consonante que la sigue, es decir, a la $f$. Dicho proceso, de acuerdo con su punto de articulación, se llama labiodentalización.

La siguiente realización que puede verse en la tabla 2, es la correspondiente a la variante interdental $/ \mathrm{n} /$. Ésta se presenta ante la consonante fricativa interdental $/ \theta /$, por ejemplo, en las palabras como onceno, encima o pinza. El proceso que se produce, originado en la zona supraglótica, se llama interdentalización.

La variante dentoalveolar / $/ \mathrm{n} /$ de la $/ \mathrm{n} /$ implosiva, de acuerdo con el proceso de mentalización, que consiste en el desplazamiento de la zona de articulación, la cual pasa a ser dental, aparece ante las oclusivas dentales $/ \mathrm{d} / \mathrm{y} / \mathrm{t} /$, por ejemplo, en las palabras onda $\mathrm{o}$ anteayer. El alófono alveolar $/ \mathrm{n} / \mathrm{se}$ realiza "en posición inicial absoluta, en posición intervocálica y en posición final absoluta, así como en contacto con otra consonante de articulación alveolar" [Sarmiento, 1997: 45]. Cabe mencionar asimismo que en posición final existe una neutralización entre $/ \mathrm{n} / \mathrm{y} / \mathrm{m} /$. Algunas palabras con la realización alveolar de este sonido son, por ejemplo, enlace o anaco. En contacto con consonantes palatales, el alófono $/ \mathrm{n} /$ suena como predorsal nasal sonoro $/ \mathrm{n} \mathrm{j} /$, por ejemplo, en las palabras cónyuge o ancho. El proceso descrito se llama palatalización, que, tal como su nombre indica, consiste en el desplazamiento de la zona de articulación hasta el paladar. Se trata de una forma palatalizada $/ \mathrm{n}^{\mathrm{j} / \mathrm{y}}$ no de la palatal $/ \mathrm{n} /$ (de la que trataremos en breve), porque es la parte anterior del predorso de la lengua la que forma la oclusión con la zona prepalatal, de ahí que el proceso consista en una ligera palatalización y no en una palatal propiamente dicha.

La variante palatal $/ \mathrm{n} /$ aparece gracias al fenómeno de despalatalización que, por otro lado, consiste en el cambio del punto de articulación desde el paladar a los alvéolos, por ejemplo, en las palabras leña o español. Observamos aquí "la pérdida de la cualidad palatal de la consonante nasal $/ \mathrm{n} /$, que pasa a articularse como alveolar o como nasal palatalizada: $/ \mathrm{n} />/ \mathrm{n} />/ \mathrm{ni} />/ \mathrm{n}^{\mathrm{j} /}$ " [Blecua, 2011: 244]. "Se realiza así en posición inicial de sílaba y en posición intervocálica. No se realiza en posición final" [Sarmiento, 1997: 46]. 
Por último, la variante velar /y/ aparece en contacto con consonantes velares $/ \mathrm{k} /, / \mathrm{g} /, / \mathrm{x} /$, por ejemplo en palabras como manga, concreto o faringe. El proceso que observamos aquí se denomina velarización [Nowikow, Szałek, 2001: 75] y consiste en el desplazamiento del punto de articulación, que pasa a ser velar. Todas las variaciones antes descritas y los procesos mencionados se insertan dentro del fenómeno de asimilación. En este sentido, se puede apreciar aquí la disociación de algunos rasgos de un segmento para luego adoptar los del segmento contiguo.

Cabe mencionar que en el presente artículo analizamos solamente las asimilaciones regresivas por adyacencia, por lo que nos interesa el hecho de cómo la consonante que aparece inmediatamente después de una nasal influye en ella y cambia su punto de articulación. Sin embargo, tenemos que ser conscientes de que en otras posiciones en la sílaba también se producen distintos cambios y que, en otras variedades del español, se pueden observar distintas realizaciones de las consonantes nasales.

\section{Las consonantes nasales en la lengua polaca}

El sistema de las consonantes nasales en polaco es igual al del español. También diferenciamos tres consonantes nasales, en las que observamos múltiples realizaciones. Las consonantes nasales básicas son las que se presentan en la siguiente tabla:

\begin{tabular}{|c|c|c|c|}
\cline { 2 - 4 } \multicolumn{1}{c|}{} & bilabial & alveolar & palatal \\
\hline sonoras nasales & $\mathrm{m}$ & $\mathrm{n}$ & $\mathrm{n}$ \\
\hline
\end{tabular}

Tabla 3. Consonantes nasales en polaco. No obstante, estas tres nasales presentan variados alófonos. Las realizaciones que se producen en el polaco podremos verlas en la tabla que se ofrece a continuación, en la cual aparecen todas las variantes de las nasales en polaco, aunque no todas serán objeto de 
análisis en el presente trabajo puesto que algunas aparecen solamente gracias a asimilaciones progresivas.

\begin{tabular}{|c|c|c|c|}
\hline \multicolumn{4}{|c|}{ nasales } \\
\hline $\mathrm{m}$ & sonora & \multirow{2}{*}{ duras } & \multirow{4}{*}{ bilabiales } \\
\hline $\mathrm{m}$ & sorda & & \\
\hline $\mathrm{m}^{\prime}$ & sonora & \multirow{2}{*}{ blandas } & \\
\hline $\mathrm{m}^{\prime}$ & sorda & & \\
\hline $\mathrm{n}$ & sonora & \multirow{2}{*}{ duras } & \multirow{3}{*}{ dentales } \\
\hline $\mathrm{n}$ & sorda & & \\
\hline $\mathrm{n}^{\prime}$ & sonoras & blandas & \\
\hline ṇ. & sonora & \multirow{2}{*}{ duras } & \multirow{2}{*}{ alveolares } \\
\hline ṇ, & sorda & & \\
\hline $\mathrm{n}$ & sonora & \multirow{2}{*}{\multicolumn{2}{|c|}{ palatales }} \\
\hline $\mathrm{n}^{\prime}$ & sorda & & \\
\hline$\eta$ & sonora & \multirow{2}{*}{ duras } & \multirow{4}{*}{ velares } \\
\hline $\mathrm{y}$. & sorda & & \\
\hline$y^{\prime}$ & sonora & \multirow{2}{*}{ blandas } & \\
\hline y.' & sorda & & \\
\hline
\end{tabular}

Tabla 4. Fragmento del cuadro de sonidos del polaco. Realizaciones de las consonantes nasales en polaco [Ostaszewska, Tambor, 2011: 34]. Es evidente que el sistema polaco presenta más realizaciones de las consonantes nasales que el sistema español. Las primeras diferencias que hemos de señalar, consisten en que en la lengua polaca se diferencian las consonantes duras y las blandas según el criterio de la existencia o ausencia de palatalización, rasgo que, por otro lado, en español no se observa. Otra diferencia es que los polacos distinguen también las consonantes sonoras de las sordas: así, en polaco hay fonemas consonánticos que, con idéntico punto de articulación, se oponen únicamente por la sonoridad. 


\section{Asimilaciones de las consonantes nasales en polaco}

En tratamiento de las asimilaciones de las consonantes nasales en polaco, será realizado paso a paso, atendiendo al orden en el que éstas han aparecido en la tabla 4. En este apartado nos centraremos no solamente en las asimilaciones regresivas por adyacencia, sino también en las asimilaciones regresivas por cercanía, es decir, en las que un fonema se pronuncia con rasgos fonéticos de un fonema cercano posterior.

$\mathrm{La} / \mathrm{m} /$ bilabial dura sonora es la realización que aparece en posición inicial de palabra o en posición intervocálica. Su realización sorda aparece en las asimilaciones progresivas, puesto que en el cambio de su sonoridad influye la consonante que la precede, por ejemplo, en la palabra rytm: la /t/ es una dental sorda y por contagio fonético, la $/ \mathrm{m} /$ también pierde su sonoridad. Para que se den las blandas, tiene que aparecer la asimilación anticipatoria por cercanía, puesto que no necesariamente en la realización de este alófono influye el segmento inmediatamente posterior, sino que también pueden ser los siguientes. Esa situación se observa en la palabra miś, donde la $/ \mathrm{m} /$ bilabial dura pasa a realizarse como blanda por la influencia de la vocal que la sigue, la /i/. La misma situación, pero con la falta de sonoridad se da en la palabra $k l a m k i$, donde la $/ \mathrm{m} /$ ha perdido su sonoridad por la influencia del alófono sordo $/ \mathrm{k} /$, y por la $/ \mathrm{i} /$, que debilita a $/ \mathrm{k} /$, influyendo al mismo tiempo en la realización de la $/ \mathrm{m} /$.

La dental dura sonora $/ \mathrm{n} /$ aparece en posición inicial de palabra o en posición intervocálica, mientras que su realización sorda se da tras el segmento sordo que la precede, por ejemplo, en las palabras snob o snajper. La realización blanda aparece ante una palatal $/ \mathrm{j} /$, por ejemplo, en pan Jan, donde la /j/ debilita la pronunciación de la /n/ dental. Las alveolares, ambas duras, se diferencian entre sí solamente por su sonoridad. La sonora /ṇ/, se puede apreciar ante el alófono /č/, por ejemplo, en la palabra poncz; mientras que la sorda se realiza también delante del mismo alófono, pero seguido de una sorda, por ejemplo en $/ \mathrm{k} /$, en la palabra garnczka. Por supuesto, articulado de 
una forma muy rápida dicho sonido nasal se pierde completamente, mientras que, pronunciando la palabra con mucho cuidado, podemos mantener su sonoridad. Cabe mencionar, hablando de las asimilaciones, que se trata de cambios fonéticos que aparecen en nuestra habla cada vez con mayor frecuencia, dado que la tendencia del hablante es a articular los sonidos más rápidamente, no prestando una razonable atención a la pronunciación.

En cuanto a las palatales, éstas se diferencian solamente por su sonoridad. El sonido / $/ \mathrm{n} /$ sonoro puede apreciarse en las palabras nie o koń. Como se puede observar, la grafía es distinta puesto que en el primer ejemplo notamos un debilitamiento de la $/ \mathrm{n} /$ por influencia de la /i/ que la sigue, mientras que en el segundo ejemplo, el alófono está representado con una sola letra [Dukiewicz, 1967: 10]. La pronunciación de ambas palabras es idéntica. Para que la consonante nasal palatal $/ \mathrm{n} /$ sea sorda, tiene que aparecer en vecindad de otra sorda. Ese sonido palatal sordo lo representa bien la palabra baśń, donde la $/ \mathrm{n}^{\prime} /$ aparece en el sonido final, por lo que normalmente en el habla pierde su sonoridad.

Las últimas realizaciones que quedan por analizar son las velares. Al igual que en el sistema español, se realizan ante otras velares $/ \mathrm{k} /$, $/ \mathrm{g} /, / \mathrm{x} /$, por ejemplo, en las palabras kongres o bongo, donde vemos la realización velar dura sonora. La variante sorda aparece cuando va seguida de una sorda, dándose entonces el caso de asimilación progresiva. Por ejemplo, por la influencia de la /s/ en la palabra pios$n k a, 1 \mathrm{a} / \mathrm{y} /$ ha perdido su sonoridad [Ostaszewska, Tambor, 2011: 86]. Para que surja el debilitamiento, en la vecindad cercana tiene que aparecer una /i/: así, en la palabra Helenki, observamos que la última /i/ influye no solamente en la velar / $\mathrm{k} /$ debilitándola, sino también en la nasal precedente. Por último, cabe añadir que para que ese sonido nasal velar sea al mismo tiempo blando y sordo, tiene que cambiarlo una sorda precedente, por ejemplo una /s/ en la palabra piosnki, donde la $/ \mathrm{y} /$ velar pierde su sonoridad. Este mismo sonido aparece en la pronunciación de las palabras que carecen de la consonante nasal pero disponen de una vocal nasal seguida de $k$ o $g$, por ejemplo en las palabras sęk, pręga, bąk [ejemplos de: Dukiewicz, 1967: 10]. 
Como acabamos de ver, tras el análisis de ambos sistemas de nasales, los procesos que hacen variar su pronunciación son muy similares. La asimilación, fenómeno muy estrechamente relacionado con el desarrollo de una lengua, es un proceso muy vivo y actual, produciéndose prácticamente en cada idioma, al asimilar un fonema a otro cercano.

\section{Divergencias en la pronunciación de las nasales en varios gru- pos consonánticos}

En este penúltimo apartado, observaremos algunos problemas de pronunciación de las nasales en diversos grupos consonánticos, así como las divergencias en la realización de las mismas en los sistemas de las nasales en las lenguas española y polaca.

En la lengua española hay muchas palabras en las que aparece un grupo consonántico con dos segmentos nasales $n m$ o $n n$. En el grupo $\mathrm{nm}$, la /n/ alveolar, de acuerdo con el proceso de bilabialización, asimila su punto de articulación a la $/ \mathrm{m} /$ bilabial, por lo que ambas consonantes tienen la misma zona de articulación. De acuerdo con las normas de la RAE del año 1992, la pronunciación de dos consonantes iguales en posición silábica interna se diferencia fonéticamente de la de una consonante simple intervocálica por su mayor duración; por eso, en las palabras conminar o conmovedor, el sonido representado por las letras $/ \mathrm{nm} /$ es realizado como si fuera solo una $/ \mathrm{m} /$ pronunciada por más tiempo [Matte Bon, 2005: 334]. Por su parte, en la lengua polaca, no hay palabras con el grupo consonántico $\mathrm{nm}$, pero existen muchas con doble $n$ o doble $m$, por ejemplo: inny, innowacja o gamma. La pronunciación actual tiende a prolongar el sonido de la $/ \mathrm{n} /$ alveolar sonora, pero es también correcto y muy frecuente realizar esa grafía pronunciando dos $n$. En cuanto a la realización de las doble $\mathrm{m}$, cabe mencionar que éstas pierden su sonoridad [Wierzchowska, 1971: 152]. 
Otra divergencia notable, se observa en el grupo consonántico ns. La RAE admite la doble forma y pronunciación de este grupo, por ejemplo, en las palabras transcender/trascender, transbordo/trasbordo. No obstante, se auspicia la reducción de ese grupo en otras palabras, por ejemplo, transmisión o transparente. En el caso de la lengua polaca, nunca se omite ese sonido nasal, dado que siempre es pronunciado por los hablantes, aunque pierde su sonoridad, por ejemplo, en transfer o transakcja.

Antes de pasar a las conclusiones finales, cabe mencionar también una peculiaridad de la lengua polaca, que, como ya hemos visto, dispone de vocales nasales. Hay muchas palabras en polaco que, aunque en su grafía poseen una consonante nasal, en su pronunciación se pierde. Esa situación puede observarse, por ejemplo, en la palabra koński, cuya pronunciación común y rápida causa la pérdida de la consonante nasal, optando por la prolongación y nasalidad de la vocal precedente. Aunque la /o/ no es una vocal nasal, por influencia de la palatal $/ \mathrm{n} /$ adquiere una vibración adicional. Hay también palabras como paczek, mąka, uragać, donde en la ortografía no aparece ninguna nasal, aunque en la pronunciación sí que se da [Wierzchowska, 1971: 136]. La vocal nasal pierde su nasalidad y se divide en dos sonidos: una vocal /o/ y una nasal /n/ alveolar [Ostaszewska, Tambor, 2011: 22]. Estos fenómenos no los encontraremos en el español porque su sistema vocálico está limitado a cinco sonidos, no disponiendo de las vocales nasales polacas.

\section{Conclusiones finales}

El contenido expuesto en el presente artículo ha intentado realizar un acercamiento del sistema de las consonantes nasales en las lenguas española y polaca. Como hemos podido apreciar, ambos sistemas son muy parecidos puesto que disponen de las mismas nasales básicas. No obstante, en cuanto a sus realizaciones, observamos al- 
gunas discrepancias, las cuales surgen porque el sistema consonántico polaco distingue entre sonidos duros y blandos así como también entre sordos y sonoros. Por esa cuestión, analizando distintas realizaciones de las nasales polacas, hemos ofrecido más ejemplos para poder dar una visión más amplia en cuanto a su pronunciación. Sin embargo, las asimilaciones observadas son las mismas, porque los aparatos articulatorios humanos son iguales, por lo que la vecindad de algunos segmentos varía la pronunciación y el punto de articulación de otros.

En el penúltimo apartado de este trabajo, dedicado a los problemas de pronunciación de algunos grupos consonánticos con una nasal o la peculiaridad polaca de disponer de las vocales nasales, nos percatamos de que la pronunciación y la corrección fonética no siempre es la misma, puesto que las reglas y normas lingüísticas son distintas.

\section{Bibliografía}

BIEDRZYCKI, L. (1963), „Fonologiczna interpretacja polskich głosek nosowych", Biuletyn Polskiego Towarzystwa Językoznawczego, 22, pp. 23-45.

BLECUA, J.M. (ed.) (2011), Nueva gramática de la lengua española. Fonética y fonología, Espasa Libros, Barcelona.

DUKIEWICZ, L. (1967), Polskie głoski nosowe, Państwowe Wydawnictwo Naukowe, Warszawa.

MARTÍNEZ CELDRÁN, E., FERNÁNDEZ PLANAS, A.M. (2007), Manual de fonética española. Articulaciones y sonidos del español, Ariel, Barcelona.

MATTE BON, F. (2005), Gramática comunicativa del español, t. I, Edelsa, Madrid.

NOWIKOW, W. (1996), Fonetyka hiszpańska, Wydawnictwo Naukowe PWN, Warszawa.

NOWIKOW, W., SZAŁEK, J. (2001), Introducción a la fonología y la fonéticaespañolas, Wydawnictwo Naukowe UAM, Poznań.

OSTASZEWSKA, D., TAMBOR, J. (2011), Fonetyka i fonologia wspótczesnego języka polskiego, Wydawnictwo Naukowe PWN, Warszawa. 
Asimilación fonética anticipatoria de las consonantes nasales...

SARMIENTO, R. (1997), Manual de corrección gramatical y de stilo, SGEL, Madrid.

WIERZCHOWSKA, B. (1971), Wymowa polska, Państwowe Zakłady Wydawnictw Szkolnych, Warszawa. 